引用格式: 周迪, 周丰年, 王雪芹. 低碳试点政策对城市碳排放绩效的影响评估及机制分析[J]. 资源科学, 2019, 41(3): 546-556. [Zhou D, Zhou F N, Wang X Q. Impact of low-carbon pilot policy on the performance of urban carbon emissions and its mechanism [J]. Resources Science, 2019, 41(3): 546-556.] DOI: 10.18402/resci.2019.03.12

\title{
低碳试点政策对城市碳排放绩效的影响评估及 机制分析
}

\author{
周 迪 ${ }^{1}$, 周丰年 $^{2}$, 王雪芹 ${ }^{3}$
}

(1. 广东外语外贸大学数学与统计学院,广州 510006;

2. 长江水利委员会长江口水文水资源勘测局, 上海 200136;

3. 广东外语外贸大学信息科学与技术学院, 广州 510006)

\begin{abstract}
摘 要: 低碳试点是实现中国低碳经济发展的一项重要政策,客观评价其实施效果,不仅有利于低碳试点地区 更好地推进低碳工作, 而且对于低碳试点政策的进一步推广具有重要意义, 但低碳试点政策能否实现碳排放绩效 的提升尚未得出一致的结论。本文以第二批低碳试点政策为例,基于2012-2016年的地级市面板数据,采用倾向 得分匹配一双重差分(PSM-DID) 方法研究低碳试点政策对降低城市碳排放强度的影响, 以有效降低样本自选择及 政策内生性等问题导致的处理效应偏差。研究发现: 低碳试点政策对城市的碳排放强度下降具有显著且持续的推 动作用; 进一步的机制识别结果显示, 低碳试点城市主要是通过能源效率的提高以及产业结构升级等方式实现碳 强度的下降, 且产业升级的效果有逐年增强的趋势, 但通过城市绿地碳汇水平的提高而降低碳排放的目标尚未实 现。基于此结论, 本文认为中国应进一步推广低碳试点政策,积极探索低碳城市的发展模式,特别是提升宜居绿色 的城市环境。
\end{abstract}

关键词: 低碳试点; 碳强度;倾向得分匹配; 双重差分; 低碳城市

DOI :10.18402/resci.2019.03.12

\section{1 引言}

伴随着经济的持续发展以及化石能源的不断 消耗,全球气候变化问题引起了科学界、各国政府 和国际社会的普遍关注和重视, 中国政府为积极应 对气候变化而大力推进绿色低碳发展。2010年 7 月,国家发改委发布了《关于开展低碳省区和低碳 城市试点工作的通知》, 首批试点地区包括广东、辽 宁等五省和天津、重庆等八市。2012年11月又发布 了《国家发展改革委关于开展第二批低碳省区和低 碳城市试点工作的通知》, 确定北京、上海、海南、石 家庄等 29 个省市为第二批低碳试点地区。且通知
中还明确了试点目标, 包括申报省市要明确本地区 在“十二五”期间降低单位国内生产总值的二氧化 碳排放、非化石能源占一次能源比重以及森林碳汇 等。这些试点地区是否采取了有效措施而降低了 碳排放强度呢? 已有研究并没有得出一致的结论, 且往往重政策效应检验而忽视政策机制分析，低碳 试点地区若实现了碳排放强度的下降, 只有进一步 挖掘其背后的具体举措, 才能借助先行先试的契机 更好地发挥示范带头作用,并为其他地区提供经验 借鉴。为此, 本文尝试对这一问题展开研究, 以第 二批低碳试点地区为例,采用倾向得分匹配一双重

收稿日期: 2018-08-26 修订日期: 2018-12-05

基金项目 : 广东省自然科学基金项目(2018A030310044); 全国统计科学研究项目(2017LY55); 广东省软科学项目 (2015A070703019; 2016A070705058)。

作者简介: 周迪,男,湖北鄂州人,博士,讲师,主要研究方向为区域可持续发展, 区域资源环境。E-mail: zhoudi19880101@163.com 通讯作者:周丰年,E-mail: cjkfnzhou@126.com 
Matching, PSM)进行样本匹配, 再在匹配后的样本

差分方法进行实证分析, 为更好地发展低碳经济提 供政策参考。

随着中国低碳试点工作的不断推进, 对低碳试 点工作的相关研究也成为学术界的热点, 梳理相关 文献发现,该项研究主要从以下几个方面展开:第 一, 结合低碳试点省市, 对中国低碳试点政策以及 低碳试点地区的低碳发展展开探讨, 主要从发展特 点、发展现状以及发展路径等方面进行研究 ${ }^{[1-6]}$; 第 二, 对低碳试点省市的低碳发展绩效进行评价, 主 要从碳排放水平、碳排放强度等方面进行考察和评 价 ${ }^{[7-9]}$ 。这类研究往往从低碳试点地区本身出发, 根 据其在试点前后碳排放绩效的变化来进行政策评 价, 属于 “单差分析” 范畴。第三, 通过对试点及非 试点地区的比较来评价低碳试点政策效应。与第 二类研究无法得到低碳试点政策的净效应不同,这 类研究往往采用当前较为流行的政策评价方法。 如邓荣荣等 ${ }^{[10]}$ 以中国首批低碳试点的 8 个城市为实 验组,采用双重差分 (Difference in Difference,DID) 方法研究低碳试点政策对试点城市碳排放绩效的 影响, 结果发现有积极的政策效应, 且政策效应随 着试点年限的增长逐步增强; 李顺毅 ${ }^{\left[{ }^{11]}\right.}$ 以第二批低 碳试点城市为考察对象, 采用合成控制法发现低碳 试点政策能降低城市的用电强度, 且这种政策效应 有逐年增强的特征。类似地, 戴嵘等 ${ }^{[12]}$ 以第一批试 点省份为实验组, 采用 DID 方法对低碳试点政策的 碳减排效应进行评价, 发现试点政策能显著降低人均 碳排放量。但也有研究得出相反的结论, 如冯粀 ${ }^{[13]}$ 发现低碳试点政策不仅对降低华东地区省会城市 碳强度的效果不明显, 反而有阻碍作用; 陆贤伟 ${ }^{[14]}$ 基 于省份数据对第一批低碳试点政策进行评价, 发现 在试点政策实施后, 试点与非试点地区的碳排放量 并没有显著性的差异。

可见,已有文献对低碳试点政策效果进行了较 为充分的讨论, 但也存在以下不足:第一, 已有研究 往往直接采用双重差分模型进行低碳政策效果的 实证检验, 而直接采用DID 模型可能会因为实验组 和对照组数据不满足该模型要求的随机性和同质 性假设, 从而导致研究结果的偏误。为此, 本文基 于地级层面的大样本数据优势, 首先采用 Heckman 等 ${ }^{[15]}$ 提出的倾向得分匹配方法 (Propensity Score
基础上进行 DID 检验, 以尽可能满足 DID 模型所要 求的随机性和同质性假设, 并对结论进行稳健性检 验,确保实证结果的准确; 第二,已有研究往往重在 政策效果的检验,对于政策背后的作用机制缺乏深 人探讨, 为此, 本文在政策效果基础上,进一步从多 方面探讨其作用机制。

\section{2 理论分析与研究假设}

随着中国工业化、城镇化进程的加快, 经济发 展对能源的依赖性不断增大, 且中国“富煤、少气、 缺油” 的资源条件致使能源结构只能以煤为主, 导 致碳排放量位居世界前列, 面临着巨大的碳减排压 力 $^{[16]}$ 。若低碳试点城市能合理布局低碳产业, 特别 是低能耗、低排放的第三产业, 将实现能源消耗的 降低,实现绿色发展, 最终达到生态环境和经济增 长的和谐共赢。除了产业结构升级外, Palencia 等 ${ }^{[17]}$ 认为, 发展中国家工业发展需要相应措施保证可持 续性发展,在短期提高能源效率和能源转换是降低 二氧化碳排放的有效途径,而中国改革开放以来工 业碳强度波动性下降的决定因素正是能源强度的 降低 ${ }^{[18]}$, 因此,各低碳试点城市通过改善能源效率可 实现碳强度的下降。具体从中国能源消费结构来 看, 电能消费在能源终端消费中的比重呈现不断上 升趋势, 由于现阶段仍以火力发电为主, 因而电力 的生产和消费已成为中国碳排放的重要来源。为 降低碳排放强度, 除优化电力生产结构之外, 从节 能的角度看, 有效降低电能消费强度 (即单位 GDP 耗电量) 是控制碳排放的重要基础 ${ }^{[1]}$ 。除减少碳源 外,降低大气中的二氧化碳浓度的另一个重要措施 是增加碳汇功能,而森林、灌丛和草地等绿地承担 着碳汇的重任, 因此在区域绿地的规划建设中,引 人 C4 植物能使绿地发挥最大的绿色 GDP 功效 ${ }^{[19]}$ 。 尤其是低碳试点城市应加强城市绿地碳汇功能, 这 样既可促进城市的低碳经济发展, 又能提高城市居 民的生活质量。

此外, 从政策层面上看,发改委也要求低碳试 点地区开展调查研究, 明确试点思路, 发挥规划综 合引导作用。将调整产业结构、优化能源结构、节 能增效、增加碳汇等工作相结合, 明确提出本地区 控制温室气体排放的行动目标、重点任务和具体措 
施, 降低碳排放强度, 积极探索低碳绿色发展模 式。按照低碳省市试点工作的要求, 各试点地区应 在以下六个方面开展具体的工作: 1)制定并完善低 碳发展规划; 2探索建立低碳发展体制机制的配套 体系; (3)加大产业结构调整, 初步建立低碳产业体 系; (4)探索建立温室气体数据统计、核算和管理体 系; (5)建立低碳评估考核机制; (6)树立低碳消费和低 碳生活理念 ${ }^{[19]}$ 。根据上述要求, 低碳试点省市结合 自身实际, 找准了着力点和突破口, 并开展了一系 列的工作 ${ }^{[2]}$, 在低碳发展方面取得良好的成效和经 验 ${ }^{[20]}$ 。因此, 本文认为低碳试点城市可能从产业结 构升级、节能增效、增加碳汇等方面取得一定的成 效, 从而提升碳排放绩效, 并提出如下四点研究假 设:

$\mathrm{H}_{1}$ : 低碳试点政策能提升试点地区的碳减排绩 效、降低其碳排放强度。

$\mathrm{H}_{2}$ : 低碳试点地区通过产业结构升级提升碳排 放绩效。

$\mathrm{H}_{3}$ : 低碳试点地区通过节能增效提升碳排放 绩效。

$\mathrm{H}_{4}$ : 低碳试点地区通过绿地碳汇提升碳排放 绩效。

\section{3 模型构建与变量描述}

\section{1 样本说明}

国家发展改革委于 2010 年发布的首批低碳试 点地区包括广东、辽宁、湖北、陕西、云南五省和天 津、重庆、深圳、厦门、杭州、南昌、贵阳、保定八市; 2012 年 11 月《国家发展改革委关于开展第二批低碳 省区和低碳城市试点工作的通知》中又确定北京、 上海、海南、石家庄、秦皇岛、晋城、呼伦贝尔、吉林 市、大兴安岭地区、苏州、淮安、镇江、宁波、温州、池 州、南平、景德镇、赣州、青岛、济源、武汉、广州、桂 林、广元、遵义、昆明、延安、金昌、乌鲁木齐等 29 个 省市作为第二批低碳试点省市。考虑到以城市为 单位更有代表性和典型性, 并可以在更细致的范围 内探讨低碳试点的政策效果, 同时还可避免实证分 析受样本量的限制, 本文选择第二批低碳试点城市 作为政策评价的实验组。由于两次政策中试点地 区有一些交叉, 为避免第一次试点政策的干扰, 本
文将广东、辽宁、湖北、陕西、云南、海南六省中的所 有地级市以及天津、重庆、厦门、杭州、南昌、贵阳、 保定七市剔除。另外, 考虑到北京、上海等城市和 其他城市的差异性较大不利于数据的匹配，也予以 剔除; 大兴安岭地区和济源市由于数据缺失也被剔 除。最后纳人倾向得分匹配一双重差分 (PSMDID) 分析的地区为 202 个地级市, 其中实验组和对 照组分别为 20 个和 182 个。

\section{2 模型构建}

由于低碳试点政策冲击可以看成准自然实验, 本文采用了经典的政策评价模型——双重差分模 型,该模型通过比较实验组和对照组政策冲击前后 变化的差异来量化政策的效果,但该方法在使用过 程中对于对照组和实验组有一定的要求, 即需满足 平行趋势,在政策冲击之前发展的态势一致。如果 本身的发展就有差异,则得出的差分结果会对政策 效果产生偏差。为此,本文首先采用 PSM 方法对实 验组匹配相近似的对照组, 以尽可能满足两组样本 的随机性和同质性假设, 避免样本的选择性偏差。 在匹配后的样本基础上,再采用双重差分方法进行 政策效应检验。为了对 $\mathrm{H}_{1}$ 进行检验,基本模型为:

$$
\begin{aligned}
Y_{i t} & =\beta_{0}+\beta_{1} \text { Treat }_{i t} \times T+\lambda \sum \text { Control }_{i t} \\
& +u_{i}+v_{t}+\varepsilon_{i t}
\end{aligned}
$$

式中: $Y_{i t}$ 代表 $i$ 城市第 $t$ 年的碳排放强度; Treat 用于 区分实验组和对照组, 取 1 表示是实验组,取 0 表示 为对照组; $T$ 为政策时间变量,政策发生之后的年份 取 1 , 政策当年及之前的年份取 0 ; 交乘项 $T r e a t \times T$ 是 考察低碳试点政策是否提升城市碳排放绩效的核 心解释变量, 本文主要关注其系数 $\beta_{1}$, 若其为负且显 著, 表明低碳试点政策能显著地降低低碳试点城市 的碳排放强度; Control 为一系列控制变量; $u$ 和 $v$ 分 别控制城市个体固定效应和时间固定效应; $\varepsilon$ 为随机 扰动项; $\beta_{0}$ 为截距项。

为了检验 $\mathrm{H}_{2}-\mathrm{H}_{4}$, 识别低碳试点地区碳排放绩效 提升的具体机制, 构造如下模型:

$$
\text { Mech }_{i t}=\beta_{0}+\beta_{1} \text { Treat }_{i} \times T_{t}+u_{i}+v_{t}+\varepsilon_{i t}
$$

式中: 被解释变量 Mech 分别为碳排放绩效提升的 机制变量, 即本文 $\mathrm{H}_{2}-\mathrm{H}_{4}$ 中的产业结构、能源效率以 及城市绿地碳汇水平等变量。再一次通过估计 DID 
模型, 考察低碳试点地区是否在这些机制变量上相 比非试点地区有显著性改善, 以检验假设 $\mathrm{H}_{2}-\mathrm{H}_{4}$ 。

\section{3 变量选择}

本文选取各城市的碳排放强度的对数值 (Inicar) 来衡量各城市的碳排放绩效,各城市碳排放的测算 借鉴韩峰等 ${ }^{[21]}$, 根据电力、天然气、液化石油气等能 源消耗推算出各城市的碳排放水平,计算公式为:

$$
I=C_{\mathrm{n}}+C_{\mathrm{p}}+C_{\mathrm{e}}=k E_{\mathrm{n}}+\gamma E_{\mathrm{p}}+\varphi\left(\eta \times E_{\mathrm{e}}\right)
$$

式中: $I$ 表示城市碳排放量; $C_{n} 、 C_{\mathrm{p}} 、 C_{\mathrm{e}}$ 分别表示城市 因消耗天然气、液化石油气和电力产生的碳排放 量; $E_{\mathrm{n}} 、 E_{\mathrm{p}}$ 和 $E_{\mathrm{e}}$ 分别为城市对天然气、液化石油气和 电力的消耗量; $k$ 、 $\gamma$ 分别为天然气和液化石油气的 $\mathrm{CO}_{2}$ 排放系数 ${ }^{1)} ; \varphi$ 为煤电燃料链温室气体排放系 数,折合等效 $\mathrm{CO}_{2}$ 为 $1.3203 \mathrm{~kg} /(\mathrm{kW} \cdot \mathrm{h}), \eta$ 为煤电发 电量占总发电量的比重 ${ }^{2)}$, 数据来源于《中国电力年 鉴》 $\rangle^{[22]}$ 。

交乘项 Treat $\times T$ 为本文的核心解释变量。其中 $T$ 为低碳试点政策时间的虚拟变量, 2012 年之后记 为 1,2012 年及之前记为 0 ; Treat 为是否是低碳试点 城市的虚拟变量, “是”记为 1 , “否”记为 0 。故对某 一城市而言, 若为低碳试点城市, 且 $T>2012$, 此时该 城市对应的虚拟变量 $T r e a t \times T$ 记为 1 , 否则都为 0 ; 系 数 $\beta_{1}$ 就是双重差分估计量, 代表设为低碳试点城市 政策的净效应。

根据已有研究发现, 碳排放绩效与能源强度、
产业结构以及地区的经济发展水平有着重要的关 系 ${ }^{[23-24]}$ 。为此, 本文选取人均 GDP $(\ln r g d p)$ 、能源强 度 (Inene) 以及产业结构 (Insec) 作为模型 (1) 的控制 变量。另外,选取产业结构、能源强度和碳汇水平 (Ingre) 作为机制变量对 $\mathrm{H}_{2}-\mathrm{H}_{4}$ 进行检验。具体地, 产业结构分别用第二、三产业增加值占 GDP 的比 值、能源强度用单位GDP 用电量、碳汇水平用建成 区绿化覆盖率来衡量, 具体含义见表 1 。

此外,由于PSM 匹配度本质上是用匹配变量对 政策虚拟变量做回归,将多元变量压缩成单维的倾 向得分 (条件概率), 进而根据倾向得分来匹配。因 此为了得到最佳的匹配效果, 本文的匹配变量在控 制变量基础上加人其他可能对政策选择有影响的 变量, 如城市职工平均工资 (lnwage)、城市年末总人 口 (lnpop) 衡量城市人均财富和人口规模。

\section{4 数据来源}

以上数据来源于历年《中国统计年鉴》 ${ }^{[25]}$ 《中国 城市统计年鉴》 ${ }^{[26]}$ 及国泰安数据库 ${ }^{[27]}$, 部分缺失值采 用插值法补齐。为了消除价格影响, 各城市 GDP 以 及职工平均工资分别用 GDP 指数和 GDP 平减指数 调整为实际值, 其中 GDP 指数来自各省市统计年 鉴, GDP 平减指数采用名义 GDP 和实际 GDP 相除 得到。各变量的含义及描述如表 1 所示。从表 1 可 见, 实验组和对照组之间存在着明显的差异,这为 后续研究提供了重要基础。

表 1 各变量的符号和定义

Table 1 Definition of variables

\begin{tabular}{|c|c|c|c|c|c|c|c|}
\hline \multirow{2}{*}{ 变量 } & \multirow{2}{*}{ 含义 } & \multicolumn{2}{|c|}{ 全部样本 $(N=202)$} & \multicolumn{2}{|c|}{ 实验组样本 $(N=20)$} & \multicolumn{2}{|c|}{ 对照组样本 $(N=182)$} \\
\hline & & 均值 & 标准差 & 均值 & 标准差 & 均值 & 标准差 \\
\hline lnicar & 碳排放强度, 碳排放量与实际 GDP之比 & 6.256 & 0.778 & 6.325 & 0.758 & 6.248 & 0.781 \\
\hline $\ln r g d p$ & 人均 GDP, 实际 GDP/年末总人口 & 10.311 & 0.674 & 10.578 & 0.683 & 10.282 & 0.667 \\
\hline lnene & 能源强度, 全社会用电量/实际 GDP & 1.522 & 0.804 & 1.585 & 0.786 & 1.515 & 0.806 \\
\hline lnsec & 第二产业比重, 第二产业增加值/GDP & 3.880 & 0.221 & 3.856 & 0.150 & 3.883 & 0.227 \\
\hline lnthi & 第三产业比重,第三产业增加值/GDP & 3.617 & 0.228 & 3.722 & 0.202 & 3.605 & 0.228 \\
\hline lngre & 建成区绿化覆盖率/\% & 3.642 & 0.365 & 3.715 & 0.168 & 3.634 & 0.379 \\
\hline lnwage & 职工平均工资 & 10.481 & 0.350 & 10.605 & 0.247 & 10.467 & 0.357 \\
\hline lnрор & 年末总人口 & 5.894 & 0.711 & 5.934 & 0.740 & 5.890 & 0.708 \\
\hline
\end{tabular}

注: $N$ 为样本量。

1) 天然气的 $\mathrm{CO}_{2}$ 排放系数为 $2.1622 \mathrm{~kg}-\mathrm{CO}_{2} / \mathrm{m}^{3}$, 液化石油气的 $\mathrm{CO}_{2}$ 排放系数为 $3.1013 \mathrm{~kg}-\mathrm{CO}_{2} / \mathrm{m}^{3}$

2） $2012-2016$ 年煤电发电量在总发电量中的平均比例分别为 $78.7 \% 、 78.6 \% 、 75.4 \% 、 73.7 \% 、 71.9 \%$ 。 


\section{4 实证分析}

\section{1 倾向得分匹配处理}

根据本文的设计, 在进行 PSM 时, 选择两类城 市作为分析对象,将 2012年开始实施低碳试点政策 的 20 个地级市作为实验组, 在 2012 年未实施低碳 试点政策, 且不受到第一次试点政策影响的城市为 对照组,共计 202 个。本文采用人均 GDP ( $\ln r g d p)$ 、 能源强度 (lnene)、职工平均工资 (lnwage)、年末总 人口 (lnpop)、第二产业比重 (lnsec) 和第三产业比重 (lnthi) 等 6 个可观测变量对实验组和对照组城市进 行匹配。具体为: 基于上述匹配指标, 采用Probit模 型估计倾向得分, 并采用核匹配法 (Kernel Matching)确定权重,施加“共同支持”(Common Support) 条件, 最后从对照组中匹配出 116 个概率接近的城 市, 另外 66 个城市未匹配成功, 将其剔除。PSM要 求匹配后实验组和对照组在可观测变量上不存在 显著性差异,否则表明匹配所选择的方法不恰当， 核匹配估计无效。为了检验本文的匹配效果, 表 2 给出了倾向得分匹配前后所有变量的平衡性检 验。在匹配前, 实验组与对照组的人均 GDP、职工 平均工资以及第三产业比重分别在 10\%、1\%和 5\% 水平下存在显著性的差异; 匹配后, 上述 3 个变量在 实验组和对照组之间的差异显著下降, 且所有变量 都不存在显著性差异。

因此, 可以认为本文所选取的可观测变量合适
且匹配方法恰当,核匹配估计可靠。此时,实验组 和对照组城市在 2012 年具有一致的特征,且实施低 碳试点政策的概率相近, 从而满足可比性的要求, 帮助解决 DID 中实验组和对照组在政策前不完全 满足共同趋势假设所带来的问题 ${ }^{[28]}$ 。也有效剔除了 政策评估过程中的自选择问题及其他内生性问题, 使处理效应结果更可信 ${ }^{[29]}$ 。

\section{2 双重差分分析}

\subsection{1 双重差分的平行趋势双}

为准确地识别低碳试点政策对城市碳排放绩 效的推动作用,在估计双重差分模型之前, 本文考 察样本是否满足平行趋势假设, 即在低碳试点政策 之前, 实验组和对照组的碳排放强度趋势是否一 致。为此,本文绘制出 2009-2016年实验组城市和 对照组城市的平均碳排放强度对数值, 得到如图 1 所示的趋势图。

图 1 中的虚线左侧 , 即在低碳试点政策出台之 前, 实验组和对照组的碳排放强度都呈现稳定的下 降趋势, 且趋势基本一致,表明基本满足平行趋势; 在虚线的右侧, 即在政策实施后, 实验组和对照组 的碳排放强度趋势开始出现差异, 前者的碳排放强 度下降幅度大于后者。这一结果表明, 本文通过 PSM 匹配后的对照组与实验组可以满足平行趋势 假设,同时低碳试点政策能引起试点城市的碳排放 强度发生更大幅度的下降。

表 2 倾向得分匹配前后各变量的平衡性检验

Table 2 Balance test of variables before and after propensity score matching

\begin{tabular}{|c|c|c|c|c|c|}
\hline 变量 & 匹配前后 & 实验组均值 & 对照组均值 & $t$ 值 & $P$ 值 \\
\hline \multirow[t]{2}{*}{$\ln r g d p$} & 匹配前 & 10.396 & 10.094 & $1.950 *$ & 0.053 \\
\hline & 匹配后 & 10.396 & 10.341 & 0.340 & 0.755 \\
\hline \multirow[t]{2}{*}{ Inene } & 匹配前 & 1.639 & 1.607 & 0.170 & 0.864 \\
\hline & 匹配后 & 1.639 & 1.586 & 0.220 & 0.826 \\
\hline \multirow[t]{2}{*}{ lnwage } & 匹配前 & 10.384 & 10.223 & $2.660 * * *$ & 0.008 \\
\hline & 匹配后 & 10.387 & 10.367 & 0.260 & 0.794 \\
\hline \multirow[t]{2}{*}{ lnpop } & 匹配前 & 5.926 & 5.882 & 0.260 & 0.794 \\
\hline & 匹配后 & 5.926 & 5.881 & 0.200 & 0.841 \\
\hline \multirow[t]{2}{*}{ Insec } & 匹配前 & 3.909 & 3.948 & -0.770 & 0.441 \\
\hline & 匹配后 & 3.909 & 3.920 & -0.200 & 0.844 \\
\hline \multirow[t]{2}{*}{$\ln t h i$} & 匹配前 & 3.646 & 3.497 & $2.540 * *$ & 0.015 \\
\hline & 匹配后 & 3.646 & 3.621 & 0.360 & 0.741 \\
\hline
\end{tabular}

注: ***、**、分别表示在 $1 \% 、 5 \% 、 10 \%$ 的水平上显著。 
是否加人其他控制变量, 交互项 $T r e a t \times T$ 的系数都在

在上述分析的基础上,本文采用面板固定效应 模型对式 (1)进行 DID 检验, 由于固定效应估计中 对数据进行了一阶差分, 政策虚拟变量 Treat 被删 去,但这不影响估计结果及其有效性。表 3 的列 (1) 和列 (2)为面板 DID 估计结果, 其中列 (1) 没有加人 控制变量, 列(2)加人了控制变量。可以看到, 无论

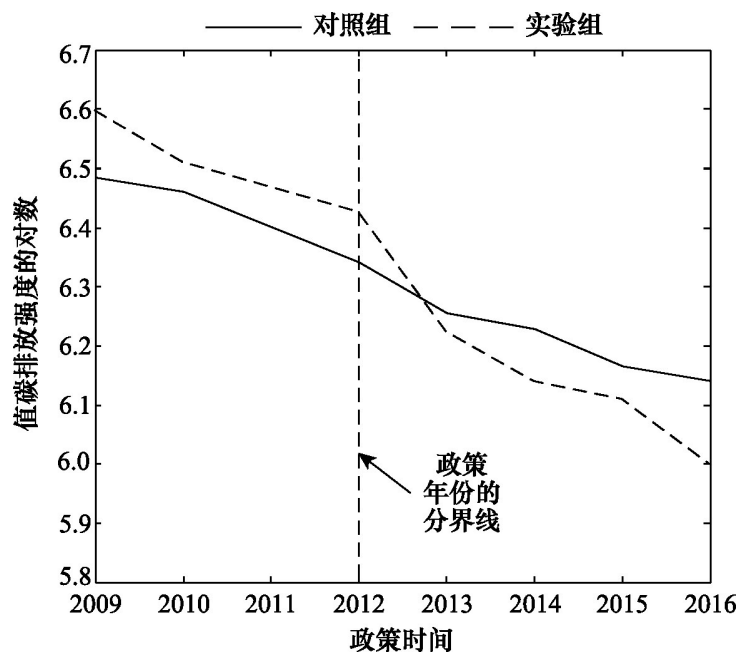

图 1 低碳试点政策前后碳排放强度的变化趋势

Figure 1 Trend of carbon emission intensity before and after implementing the low carbon pilot policy
$1 \%$ 的显著性水平下显著为负, 列 (2)在加人控制变 量后系数几乎没有发生变化。因此,列 (1) 和列 (2) 的结果与假设 1 的预期相同,低碳试点政策显著性 地降低了低碳试点城市的碳强度, 在 DID 方法下, 该结论有效地克服了政策的内生性问题,也证明了 假设 1 。

在控制变量方面, 能源强度对碳排放强度具有 显著的正影响,第二产业比重对碳排放强度也有正 向推动作用,这些结论与已有采用省级层面数据的 研究结果一致 ${ }^{[22]}$ 。

\subsection{3 动态边际影响效应}

为了进一步考察低碳试点政策对城市碳排放 绩效的动态边际影响, 在式 (1) 中加人各年的虚拟 变量 Treat $_{i t} \times T_{i j}$, 其中 $T_{i j}$ 为 2013-2016 年的时间虚拟 变量。具体的模型设定如式(4)所示, 本文主要关 心交互项 Treat $_{i j} \times T_{i j}$ 的系数 $\beta_{1}$ 。

$$
\begin{aligned}
Y_{i t} & =\beta_{0}+\beta_{1} \sum \text { Treat }_{i t} \times T_{i j}+\lambda \sum \text { Control }_{i t} \\
& +u_{i}+v_{t}+\varepsilon_{i t}
\end{aligned}
$$

式(4)的估计结果见表 3 的列 (3) 和列 (4), 可以看 到, Treat $\times T$ 的系数都在 $1 \%$ 的显著性水平下显著为 负,表明 2012 年出台低碳试点政策后,在随后的

表 3 DID 估计结果

\begin{tabular}{|c|c|c|c|c|}
\hline & \multicolumn{2}{|c|}{ 平均处理效应 } & \multicolumn{2}{|c|}{ 动态边际效应 } \\
\hline & (1) & (2) & (3) & (4) \\
\hline Treat $\times T$ & $-0.690 * * *(-6.019)$ & $-0.693 * * *(-6.483)$ & & \\
\hline$t$ & $-0.0457(-0.846)$ & $-0.0845(-0.618)$ & & \\
\hline Treat $\times t_{2013}$ & & & $-0.592 * * *(-4.134)$ & $-0.579 * * *(-4.361)$ \\
\hline Treat $\times t_{2014}$ & & & $-0.425 * * *(-2.967)$ & $-0.430^{* * *}(-3.239)$ \\
\hline Treat $\times t_{2015}$ & & & $-0.968 * * *(-6.760)$ & $-0.964 * * *(-7.265)$ \\
\hline Treat $\times t_{2016}$ & & & $-0.774 * * *(-5.402)$ & $-0.820^{* * *}(-6.194)$ \\
\hline Inene & & $0.756 * * *(9.041)$ & & $0.762 * * *(9.249)$ \\
\hline $\ln r g d p$ & & $-0.071(-0.195)$ & & $-0.219(-1.188)$ \\
\hline Insec & & $0.963 * * *(2.691)$ & & $0.860 * * *(2.838)$ \\
\hline cons & $6.421 * * *(176.913)$ & $9.740 * * *(2.612)$ & $6.421 * * *(179.036)$ & $10.846^{* * *}(3.984)$ \\
\hline 城市固定 & 是 & 是 & 是 & 是 \\
\hline 时间固定 & 是 & 是 & 是 & 是 \\
\hline$N$ & 680 & 680 & 680 & 680 \\
\hline$R^{2}$ & 0.085 & 0.210 & 0.111 & 0.237 \\
\hline$F$ & $9.950 * * *$ & $17.800^{* * *}$ & $8.370 * * *$ & $16.590 * * *$ \\
\hline
\end{tabular}

Table 3 Difference in difference (DID) estimation results

注: 括号中的值为双尾检验 $t$ 值, ***、**、分别表示在 $1 \% 、 5 \% 、 10 \%$ 的水平上显著。 
2013-2016年, 这些低碳试点城市的碳排放强度相 比非试点城市都有显著的下降, 政策的动态边际效 应持续显著。从系数的具体值可以看到, 这种动态 边际效果具有一定的波动性, 但整体呈现出明显的 上升趋势。

\subsection{4 稳健性检验}

低碳试点政策效应考察以 2013 年作为政策起 点, 考察期为 2012-2016年。为了检验实证结果的 稳健性,借鉴何靖 ${ }^{[30}$ 的思路,通过改变实验组和对照 组的考察时期来进行稳健性检验。分别将考察时 期更换为 2012-2013 年、2012-2014 年以及 2012-2015 年共3 个时间段, 同样采用 PSM-DID 方 法, 根据模型 (1)估计出平均处理效应, 具体结果见 表 4。可以发现,不管在哪个时段,也不管是否加人 控制变量, Treat $\times T$ 的系数都为负, 且都在 $1 \%$ 水平上 显著,表明本文的结果稳健。

\section{3 低碳试点政策提升城市碳排放绩效的机制分析}

低碳试点政策能够有效持续地提升碳排放绩 效, 那么低碳试点政策促进各低碳试点城市碳排放 绩效提升的机制是什么呢? 为了验证 $\mathrm{H}_{2}-\mathrm{H}_{4}$, 本文选
择第二、三产业比重、单位 GDP 耗电量及城市建成 区绿化覆盖率等变量作为因变量, 利用模型 (2)及 模型 (4)中的处理方法评估低碳试点政策的作用机 制,对于每一类因素都进行了平均处理效应和动态 边际效应的考察,得到结果见表 5。表 5列(1)、(2) 与列 (3)、(4)分别从第二和第三产业比重识别低碳 试点政策提升碳排放绩效的机制, 发现低碳试点城 市的第二产业比重有更显著的降低，同时第三产业 比重则有更显著的提升。可见，低碳试点城市在低 碳试点政策出台后,积极落实试点的要求,在产业 结构升级上取得了显著的进展。且从列 (2) 和列 (4)可以看到,产业结构效应的动态边际效应具有 递增的趋势,在政策实施后的第一年,低碳试点城 市的产业升级效果不太明显,第二产业比重下降不 显著,第三产业比重也只在 $10 \%$ 的显著性水平下提 升;但在之后年份效果日益明显,不仅都在 $1 \%$ 水平 上显著, 而且从系数来看, 升级程度也在不断增大, 假设 2 通过了检验。表 5 列 (5) 和列 (6) 给出了低碳 试点政策对城市能源强度的影响, 发现平均处理效 应为负,且在 $1 \%$ 水平上显著,可见低碳试点政策对

表 4 不同时间段下的 DID 估计结果

Table 4 Difference in difference (DID) estimation results in different time periods

\begin{tabular}{|c|c|c|c|c|c|c|}
\hline & \multicolumn{2}{|c|}{ 2012-2013年 } & \multicolumn{2}{|c|}{ 2012-2014年 } & \multicolumn{2}{|c|}{ 2012-2015年 } \\
\hline & (1) & (2) & (3) & (4) & (5) & (6) \\
\hline \multirow[t]{2}{*}{ Treat $\times T$} & $-0.592 * * *$ & $-0.587 * * *$ & $-0.508 * * *$ & $-0.504 * * *$ & $-0.662 * * *$ & $-0.652 * * *$ \\
\hline & $(-7.444)$ & $(-7.685)$ & $(-5.546)$ & $(-5.801)$ & $(-5.998)$ & $(-6.292)$ \\
\hline \multirow[t]{2}{*}{$t$} & -0.015 & -0.053 & -0.007 & 0.028 & -0.075 & 0.001 \\
\hline & $(-0.503)$ & $(-0.414)$ & $(-0.180)$ & $(0.208)$ & $(-1.486)$ & $(0.007)$ \\
\hline \multirow[t]{2}{*}{ lnene } & & $0.605 * * *$ & & $0.601 * * *$ & & $0.704 * * *$ \\
\hline & & (3.990) & & $(5.960)$ & & $(7.500)$ \\
\hline \multirow[t]{2}{*}{$\ln r g d p$} & & 0.452 & & -0.145 & & -0.352 \\
\hline & & $(0.644)$ & & $(-0.202)$ & & $(-0.535)$ \\
\hline \multirow[t]{2}{*}{ Insec } & & 0.539 & & 0.454 & & $0.787^{*}$ \\
\hline & & $(0.568)$ & & $(0.814)$ & & (1.835) \\
\hline \multirow[t]{2}{*}{ cons } & $6.421 * * *$ & 2.938 & $6.421 * * *$ & 8.748 & $6.421 * * *$ & $12.017 *$ \\
\hline & (322.396) & $(0.466)$ & $(242.208)$ & $(1.260)$ & (189.774) & $(1.840)$ \\
\hline 城市固定 & 是 & 是 & 是 & 是 & 地区 & 是 \\
\hline 时期固定 & 是 & 是 & 是 & 是 & 时期 & 是 \\
\hline$N$ & 272 & 272 & 408 & 408 & 544 & 544 \\
\hline$R^{2}$ & 0.339 & 0.411 & 0.127 & 0.230 & 0.109 & 0.222 \\
\hline$F$ & $34.310 * * *$ & $18.250 * * *$ & $13.040 * * *$ & $13.260 * * *$ & $12.290 * * *$ & $16.340 * * *$ \\
\hline
\end{tabular}

注: 括号中的值为双尾检验 $t$ 值, ***、**、*分别表示在 $1 \% 、 5 \% 、 10 \%$ 的水平上显著。 
表 5 低碳试点政策提升碳排放绩效机制的检验结果

Table 5 Mechanism test results of low carbon pilot policy to enhance carbon emission performance

\begin{tabular}{|c|c|c|c|c|c|c|c|c|}
\hline & \multicolumn{2}{|c|}{ Insec } & \multicolumn{2}{|c|}{ lnthi } & \multicolumn{2}{|c|}{ lnene } & \multicolumn{2}{|c|}{ lngre } \\
\hline & (1) & (2) & (3) & (4) & (5) & (6) & (7) & (8) \\
\hline \multirow[t]{2}{*}{ Treat $\times T$} & $-0.332 * * *$ & & $0.561 * * *$ & & $-0.331 * * *$ & & 0.056 & \\
\hline & $(-9.318)$ & & $(9.207)$ & & $(-2.807)$ & & $(1.047)$ & \\
\hline \multirow[t]{2}{*}{$t$} & $-0.068 * * *$ & & $0.091 * * *$ & & 0.009 & & 0.009 & \\
\hline & $(-4.071)$ & & $(3.149)$ & & $(0.163)$ & & $(0.457)$ & \\
\hline \multirow[t]{2}{*}{ Treat $\times t_{2013}$} & & -0.046 & & $0.115^{*}$ & & $-0.489 * * *$ & & 0.046 \\
\hline & & $(-1.265)$ & & $(1.720)$ & & $(-3.300)$ & & $(0.671)$ \\
\hline \multirow[t]{2}{*}{ Treat $\times t_{2014}$} & & $-0.213 * * *$ & & $0.440 * * *$ & & -0.143 & & 0.046 \\
\hline & & $(-5.893)$ & & $(6.581)$ & & $(-0.967)$ & & $(0.680)$ \\
\hline Treat $\times t_{2015}$ & & $-0.434 * * *$ & & $0.721 * * *$ & & $-0.530 * * *$ & & $0.128 *$ \\
\hline \multirow[t]{2}{*}{ Treat $\times t_{2016}$} & & $-0.636 * * *$ & & $0.970 * * *$ & & -0.164 & & 0.005 \\
\hline & & $(-17.622)$ & & $(14.517)$ & & $(-1.104)$ & & $(0.076)$ \\
\hline \multirow[t]{2}{*}{ cons } & $3.922 * * *$ & $3.922 * * *$ & $3.600 * * *$ & $3.600 * * *$ & $1.635 * * *$ & $1.635 * * *$ & $3.691 * * *$ & $3.691 * * *$ \\
\hline & $(347.492)$ & $(434.285)$ & (186.409) & $(215.163)$ & $(43.738)$ & (44.087) & $(217.121)$ & $(216.882)$ \\
\hline 城市固定 & 是 & 是 & 是 & 是 & 是 & 是 & 是 & 是 \\
\hline 时期固定 & 是 & 是 & 是 & 是 & 是 & 是 & 是 & 是 \\
\hline$N$ & 680 & 680 & 680 & 680 & 680 & 680 & 680 & 680 \\
\hline$R^{2}$ & 0.232 & 0.511 & 0.207 & 0.408 & 0.023 & 0.043 & 0.004 & 0.013 \\
\hline$F$ & $32.640 * * *$ & $70.090 * * *$ & $28.160 * * *$ & $46.210 * * *$ & $2.480 * *$ & $3.030 * * *$ & 0.980 & 0.850 \\
\hline
\end{tabular}

注: 括号中的值为双尾检验 $t$ 值, $* * * * * * *$ 分别表示在 $1 \% 、 5 \% 、 10 \%$ 的水平上显著。

城市能源强度下降具有显著的促进作用,但从动态 边际效应来看, 这种影响作用呈现出非连续性的特 征。在政策实施后的第一年,低碳试点城市的能源 强度相比非低碳试点城市有显著的下降,但第二年 下降程度又不显著,第三年再次呈现显著的下降， 第四年则又不显著。可见,中国低碳试点城市的能 源强度在波动中下降, 平均处理效应显著存在, 假 设 3 基本成立。表 5 列 (7)和列 (8)给出建成区绿化 覆盖率的影响效应, 结果发现不管是平均处理效应 还是动态边际效应都不显著, 双重差分面板模型的 估计结果也没有通过 $F$ 检验。这表明低碳试点政策 对城市的城市绿化率提高没有推动作用,通过碳汇 水平的显著性提升来实现碳减排的目的尚没有实 现,假设 4 未通过。

由此可见,低碳试点城市主要通过产业结构的 升级及能源强度的降低等方面提升碳排放绩效, 特 别是产业结构升级效应明显, 但城市碳汇水平未得 到显著的提高, 因此, 通过城市碳汇降低碳排放的
途径还有待进一步挖掘。

\section{5 结论与政策建议}

准确地评价低碳试点政策效果对于试点城市 更好地开展低碳试点工作, 并进一步推广低碳试点 政策具有重要意义。本文利用2012-2016年中国 202 个地级市面板数据, 运用倾向得分匹配一双重 差分方法研究了第二批低碳试点政策对城市碳排 放绩效的影响。结果发现: 低碳试点政策对低碳试 点城市碳排放强度的降低有显著且持续的推动作 用,该结论在进行稳健性检验后依然成立。进一步 的机制识别结果显示, 低碳试点城市主要通过提高 能源效率和产业结构升级的途径降低碳减排强度, 特别是,低碳试点政策在提升城市产业结构上的效 果不断增大, 但通过城市绿地碳汇水平的提升来降 低碳排放的目标尚未实现。

基于以上研究结论,本文得出的政策启示有:

(1)应该进一步推广低碳试点政策,积极探索 低碳城市的发展模式。一方面,低碳试点地区应进 
一步总结探索低碳发展的经验,如开发适宜的低碳 生态技术,采取更合理、更绿色的生产生活方式,包 括绿色交通、绿色建筑、绿色消费等, 以起到标杆示 范作用, 为全国全面开展低碳城市作出先行经验探 索;另一方面,政府对低碳试点地区的“低碳建设” 要进一步给予政策支持和理论指导, 形成一套清晰 有效的低碳政策体制机制, 如出台清晰的低碳城市 的建设目标、实施手段、评估指标以及验收标准, 以 便更友好的推广低碳试点政策.

(2)鉴于碳汇对低碳试点城市的碳排放绩效提 升作用不显著, 应大力倡导绿色发展理念,一方面 积极保护已有的森林植被, 另一方面要合理地规划 城市发展, 高度关注城镇化和工业化进程中的生态 环境问题, 营造宜居的城市环境。特别是一些欠发 达地区城市在探索低碳发展模式时, 应结合当地资 源禀赋, 发挥自然资源优势, 尽量避免盲目为追求 GDP 而发展高耗能、高排放的产业,树立“绿水青山 就是金山银山”的发展理念, 在保护好当地生态环 境的前提下发展经济, 推进新型城镇化建设。

\section{参考文献(References):}

[1] 丁丁, 蔡蒙, 付琳, 等. 基于指标体系的低碳试点城市评价[J]. 中 国人口·资源与环境, 2015, 25(10): 1-10. [Ding D, Cai M, Fu L, et al. Evaluation on low-carbon pilot cities based on indicators[J]. China Population Resources and Environment, 2015, 25(10): 110.]

[2] 丁丁, 杨秀. 我国低碳发展试点工作进展分析及政策建议[J]. 经 济研究参考, 2013, (43): 92-96. [Ding D, Yang X. Progress analysis and policy suggestions on low carbon development pilot work in China[J]. Review of Economic Research, 2013, (43): 92-96.]

[3] 刘佳骏, 史丹, 裴庆冰. 我国低碳试点城市发展现状评价研究 [J]. 重庆理工大学学报(社会科学), 2016, 30(10): 32-38. [Liu J J, Shi D, Pei Q B. Evaluation on the current development of lowcarbon pilot cities in China[J]. Journal of Chongqing Institute of Technology (Social Science Edition), 2016, 30(10): 32-38.]

[4] 盛广耀. 中国低碳城市建设的政策分析[J]. 生态经济, 2016, 32 (2): 39-43. [Sheng G Y. Policy analysis of the low-carbon city construction in China[J]. Ecological Economy, 2016, 32(2): 39-43.]

[5] 刘健, 王润, 孙艳伟, 等. 中国低碳试点省份发展路径研究 [J]. 中 国人口·资源与环境, 2012, 22(3): 56-62. [Liu J, Wang R, Sun Y $\mathrm{W}$, et al. Analysis of development path for low-carbon pilot provinces in China[J]. China Population Resources and Environment,
2012, 22(3): 56-62.]

[6] 欧阳慧. 基于碳减排视角的国家试点低碳城(镇)发展路径[J]. 城 市发展研究, 2016, 23(6): 15-20. [Ouyang H. Research on the development path of national pilot low-carbon city(town) based on the perspective of carbon emission reduction[J]. Urban Studies, 2016, 23(6): 15-20.]

[7] Lin J, Jacoby J, Cui S, et al. A model for developing a target integrated low carbon city indicator system: The case of Xiamen, China [J]. Ecological Indicators, 2014, 40(5): 51-57.

[8] 宋祺佼, 王宇飞, 齐晔. 中国低碳试点城市的碳排放现状[J]. 中 国人口·资源与环境, 2015, 25(1): 78-82. [Song Q J, Wang Y F, Qi Y. Study on present status of carbon emissions in China's lowcarbon pilot cities[J]. China Population Resources and Environment, 2015, 25(1): 78-82.]

[9] 邓荣荣. 我国首批低碳试点城市建设绩效评价及启示 $[J]$. 经济 纵横, 2016, (8): 41-46. [Deng R R. Performance evaluation and Enlightenment of the first batch of low carbon pilot cities in China [J]. Economic Review, 2016, (8): 41-46.]

[10] 邓荣荣, 詹晶. 低碳试点促进了试点城市的碳减排绩效吗? 基于 双重差分方法的实证[J]. 系统工程, 2017, 35(11): 68-73. [Deng R R, Zhan J. Does two low carbon pilot policy promote the performance of carbon emissions reduction in pilot cities: Based on difference in difference method[J]. Systems Engineering, 2017, 35 (11): 68-73.]

[11] 李顺毅. 低碳城市试点政策对电能消费强度的影响: 基于合成 控制法的分析[J]. 城市问题, 2018, (7): 38-47. [Li S Y. The impact of low carbon city pilot policy on electricity consumption intensity: Based on synthetic control method[J]. Urban Problems, 2018, (7): 38-47.]

[12] 戴嵘, 曹建华. 中国首次“低碳试点”政策的减碳效果评价: 基于 五省八市的 DID 估计 [J]. 科技管理研究, 2015, 35(12): 56-61. [Dai R, Cao J H. The effect of China's first low-carbon pilot: DID estimation of five cities and eight provinces[J]. Science and Technology Management Research, 2015, 35(12): 56-61.]

[13] 冯粀. 低碳试点城市项目对碳强度的影响评估[J]. 云南民族大 学学报(自然科学版), 2017, 26(2): 174-178. [Feng T. Assessment of the impact of pilot low-carbon city projects on carbon intensity[J]. Journal of Yunnan Nationalities University(Natural Science Edition), 2017, 26(2): 174-178.]

[14] 陆贤伟. 低碳试点政策实施效果研究: 基于合成控制法的证据 [J]. 软科学, 2017, 31(11): 98-101. [Lu X W. Study on the effectiveness of the low carbon policy: Evidence from the synthetic control methods[J]. Soft Science, 2017, 31(11): 98-101.]

[15] Heckman J J, Ichimura H, Todd P E. Matching as an econometric evaluation estimator: Evidence from evaluating a job training programme[J]. Review of Economic Studies, 1997, 64(4): 605-654. 
基于 1995-2009年省际面板数据的研究[J]. 数量经济技术经 济研究, 2012, 29(5): 36-49. [Yang Q, Liu H J. Decomposition of regional differences in carbon dioxide emissions in China and its influencing factors: A study based on interprovincial panel data from 1995 to 2009[J]. The Journal of Quantitative \& Technical Economics, 2012, 29(5): 36-49.]

[25] 中华人民共和国国家统计局. 中国统计年鉴[M]. 北京: 中国统 计出版社, 2013-2017. [National Bureau of Statistics of the People's Republic of China. China Statistical Yearbook [M]. Beijing: China Statistics Press, 2013-2017.]

[26] 中国国家统计局城市社会经济调查司. 中国城市统计年鉴[M] 北京: 中国统计出版社, 2013-2017. [Department of Urban Socio-Economic Survey of the National Bureau of Statistics of the People's Republic of China. China's Urban Statistical Yearbook [M]. Beijing: China Statistics Press, 2013-2017.]

[27] 深圳国泰安教育技术股份有限公司. CSMAR 区域经济数据库 [EB/OL]. (2018-08-12)[2018-08-26]. http: //www. gtarsc. com/ Single Table/ Data BaseInfo? nodeid=23. [Guotaian Shenzhen Education Technology Limited by Share Ltd. CSMAR Regional Economic Database[EB/OL]. (2018- 08- 12) [2018- 08-26]. http: // www. gtarsc. $\mathrm{com} /$ SingleTable/DataBaseInfo?nodeid=23.]

[28] 刘瑞明, 赵仁杰. 西部大开发一一增长驱动还是政策陷阴: 基于 PSM-DID 方法的研究[J]. 中国工业经济, 2015, (6): 32-43. [Liu R M, Zhao R J. Western development-Growth drive or policy trap: An analysis based on PSM-DID method[J]. China Industrial Economics, 2015, (6): 32-43.]

[29] 崔嵩, 周振, 孔祥智. 父母外出对留守儿童营养健康的影响研 究: 基于 PSM 的分析[J]. 农村经济, 2015, (2): 103-108. [Cui S, Zhou Z, Kong X Z. Study on the influence of parents' outgoing on the nutrition and health of left behind children: Analysis based on PSM[J]. Rural Economy, 2015, (2): 103-108.]

[30] 何靖. 延付高管薪酬对银行风险承担的政策效应: 基于银行盈 余管理动机视角的 PSM-DID 分析[J]. 中国工业经济, 2016, (11): 126-143. [He J. The policy effect of CEO deferred compensation on bank risk taking: A PSM-DID analysis based on bank earnings management[J]. China Industrial Economics, 2016, (11): 126-143.]

[24] 杨骞, 刘华军. 中国二氧化碳排放的区域差异分解及影响因素: 


\title{
Impact of low-carbon pilot policy on the performance of urban carbon emissions and its mechanism
}

\author{
ZHOU Di', ZHOU Fengnian², WANG Xueqin ${ }^{3}$ \\ (1. School of Mathematics and Statistics, Guangdong University of Foreign Studies, Guangzhou 510006, China; \\ 2. Changjiang River Estuary Bureau of Hydrological and Water Resources Survey, \\ Changjiang River Estuary Water Environment Monitoring Center, Shanghai 200136, China; \\ 3. School of Information Science and Technology, Guangdong University of Foreign Studies, Guangzhou 510006, China)
}

\begin{abstract}
Low-carbon pilot project is an important policy to realize the development of lowcarbon economy in China. Objectively evaluating its implementation effect is not only conducive to better promoting low- carbon work in low- carbon pilot project areas, but also of great significance for the further promotion of low-carbon pilot policy. However, there is no consistent conclusion on whether the low-carbon pilot policy can enhance the performance of carbon emissions. Taking the second batch of low-carbon pilot projects as an example and using city panel data from 2012 to 2016, this study examined the impact of low-carbon pilot policy on the reduction of local carbon emission intensity by using the Propensity Score Matching-Difference in Difference (PSM-DID) method in order to effectively reduce the processing effect bias caused by the problem of sample selection and policy endogeneity. It was found that the low-carbon pilot policy had a significant and sustained effect on local carbon intensity reduction. Further mechanism identification results show that low-carbon pilot areas mainly achieve a decline in carbon intensity through the improvement of energy efficiency and upgrading of industrial structure, and the upgrading of industrial structure shows a trend of increase year by year. However, the goal of reducing carbon emissions by raising the level of carbon sink in urban green spaces has not yet been achieved. Based on this conclusion, we believe that China should further promote the lowcarbon pilot policy and actively explore the development model of low-carbon cities, in particular by further establishing a livable and green urban environment.
\end{abstract}

Key words: low-carbon pilot; carbon intensity; propensity score matching; double difference; lowcarbon cities 